\title{
Synthesis of 5-Substituted 2,9-Dimethyl-1,10-Phenanthroline Dialdehydes and Their Schiff Bases with Sulfur-Containing Amines
}

\author{
Zinia $\operatorname{Jaman}^{1}$, Mohammad R. Karim ${ }^{1 *}$, Tasneem A. Siddiquee ${ }^{1}$, \\ Aminul H. Mirza' ${ }^{2}$ Mohamad A. Ali ${ }^{2}$ \\ ${ }^{1}$ Department of Chemistry, Tennessee State University, Nashville, USA \\ ${ }^{2}$ Department of Chemistry, Faculty of Science, University Brunei Darussalam, Gadong, Brunei Darussalam \\ Email: *mkarim@tnstate.edu
}

Received July 4, 2013; revised August 14, 2013; accepted August 31, 2013

Copyright (C) 2013 Zinia Jaman et al. This is an open access article distributed under the Creative Commons Attribution License, which permits unrestricted use, distribution, and reproduction in any medium, provided the original work is properly cited.

\begin{abstract}
Eight new Schiff bases of 5-nitro and 5-bromo-substituted 1,10-phenanthroline-2,9-dicarboxaldehydes with sulfur-containing amines, thiosemicarbazide, S-alkyl/aryl dithiocarbazates and 2-mercaptoaniline have been synthesized and characterized by a variety of spectroscopic methods. The condensation reactions of the dialdehydes with the amines were carried out both in the presence and absence of conc. sulfuric acid. A significant increase in yield of the Schiff bases was observed when the reactions were carried out in the presence of sulfuric acid.
\end{abstract}

Keywords: 5-Nitro-1,10-Phenanthroline Dialdehyde; 5-Bromo-1,10-Phenanthroline Dialdehyde; Schiff Bases; S-Alkyl/Aryldithiocarbazates; Thiosemicarbazide

\section{Introduction}

Recently, nitrogen and sulfur-containing organic chelating agents such as the Schiff bases derived from 2,9-dimethyl-1,10-phenanthroline dialdehyde and sulfur-containing amines and their metal complexes have received considerable attention because of their important roles in synthetic and medicinal chemistry[1]. By properly designing this type of compounds and studying their structure-activity relationships, potentially useful antibacterial, antifungal and anticancer agents can also be synthesized [2]. 2,9-Dimethyl-1,10-phenanthroline and its derivatives from which 2,9-dimethyl-1,10-phenanthroline dialdehydes are prepared, are themselves important ligands for complexation with many metal ions [3]. This property has made them important in different areas like self-assembly and catalysis. It has also played a significant role in both analytical and preparative coordination chemistry as well as in the preparation of many mixed-ligand complexes [4].

Although a large number of Schiff bases containing "hard" nitrogen and "soft" sulfur donor atoms have been synthesized using S-alkyl/aryl dithiocarbazates and het-

${ }^{*}$ Corresponding author. erocyclic aldehydes and ketones, which are able to form stable complexes with a variety of metal ions [5], less work has been reported on Schiff bases formed by condensation of 1,10-phenanthroline dialdehydes with sufurcontaining amines such as S-alkyl/aryl dithioicarbazates, thiosemi-carbazide and aminobenzenethiol.

In view of the importance of Schiff bases derived from 1,10-phenanthroline dialdehyde and sulfur-containing amines in coordination chemistry and biology, we report here the synthesis and characterization of eight new Schiff bases formed by condensation of 5-nitro-1,10-phenanthroline-2,9-dialdehyde and 5-bromo-1,10-phenanthroline-2,9-dialdehyde with different types of sulfurcontaining amines.

\section{General Methods and Procedures}

HPLC grade solvents were used in all the reactions. The conventional method of synthesis of the Schiff bases involves refluxing the reaction mixture containing the dialdehydes and amines for 1 hour followed by filtration of the solid products using suction filtration.

In all the reactions, 2 - 3 drops of conc. sulfuric acid were used. The solid product that had formed was filtered off using suction filtration. All the NMR data 
were recorded on a $400 \mathrm{MHz}$ Varian NMR Spectrometer. Mass Spectra were obtained on a Varian LC-MS with ESI.

\section{Synthesis}

We have previously reported the preparation, characterization and X-ray structures of different types of Schiff bases derived from 1,10-phenanthroline [6]. Now we report here the synthesis and characterization of eight new Schiff bases formed by condensation of the 5bromo- and 5-nitro-substituted phenanthroline dialedhydes with amines containing thione or thiol sulfur donor atoms in their backbones. All the compounds have been structurally characterized by different spectroscopic methods.

\subsection{Synthesis of 5-Nitro-1,10-Phenanthroline- 2,9-Dicarboxaldehyde from 5-Nitro 2,9-Dimethyl-1,10-Phenanthroline}

5-Nitro-2,9-dimethyl-1,10-phenanthroline (2) was synthesized from 2,9-dimethyl-1,10-phenanthroline hemihydrate (1) following a previously reported procedure (Scheme 1) [7,8]. The yield of the compound was found to be the same as reported in the literature. The crude product was purified by chromatography using combi flash (ethyl acetate: dichloromethane) to give the pure compound [Y: 70\%].

5-Nitro-1,10-phenanthroline-2,9-dicarboxaldehyde (3) was synthesized from 5-nitro-2,9-dimethyl-1,10-Phenanthroline (2) following a known procedure (Scheme 2) [9]. The yield of the compound was found to be the same as that reported in the literature. The crude product was recrystallized from chloroform and dried under vacuum to give the pure compound [Y: $91 \%$ ].

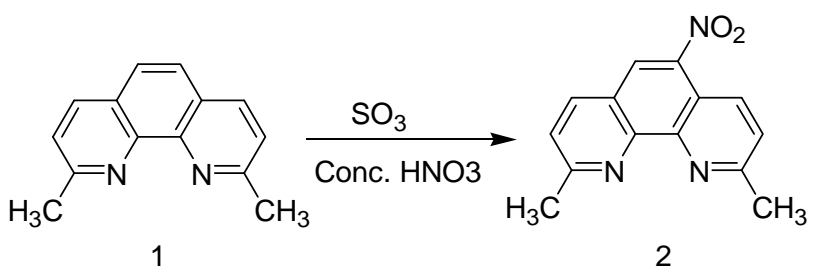

Scheme 1. Synthesis of 5-Nitro-1,10-phenanthroline.

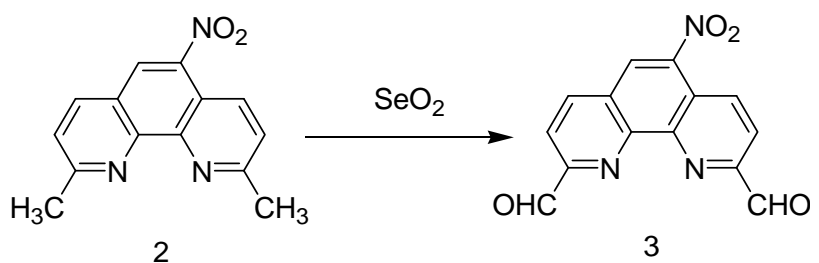

Scheme 2. Synthesis of 5-Nitro-1,10-Phenanthroline-2,9-dialdehyde.

\subsection{Synthesis of 5-Bromo-1,10-Phenanthroline- 2,9-Dicarboxaldehyde from 5-Bromo-2,9- Dimethyl -1,10-Phenanthroline}

The previously reported procedure [3] involving three steps, was also used here to synthesize 5-bromo-2,9-dimethyl-1,10-phenanthroline (6) from 2,9-dime-thyl-1, 10-phenanthroline (1) (Scheme 3).

The yield of the compound was found to be the same as that reported in the literatures procedure. [Y: 85\%].

5-Bromo-1, 10-phenanthroline-2,9-dicarboxal dehyde (7) was synthesized from 5-bromo-2,9-dimethyl-1,10phenanthroline (6) following a known procedure [10] (Scheme 4). An increase of reaction time resulted in the increase in the yield of the dialdehyde. The crude product was recrystallized from hot ethanol and dried under vacuum to give the pure compound [Y: $82 \%$ ]. The reported yield was $62 \%$.
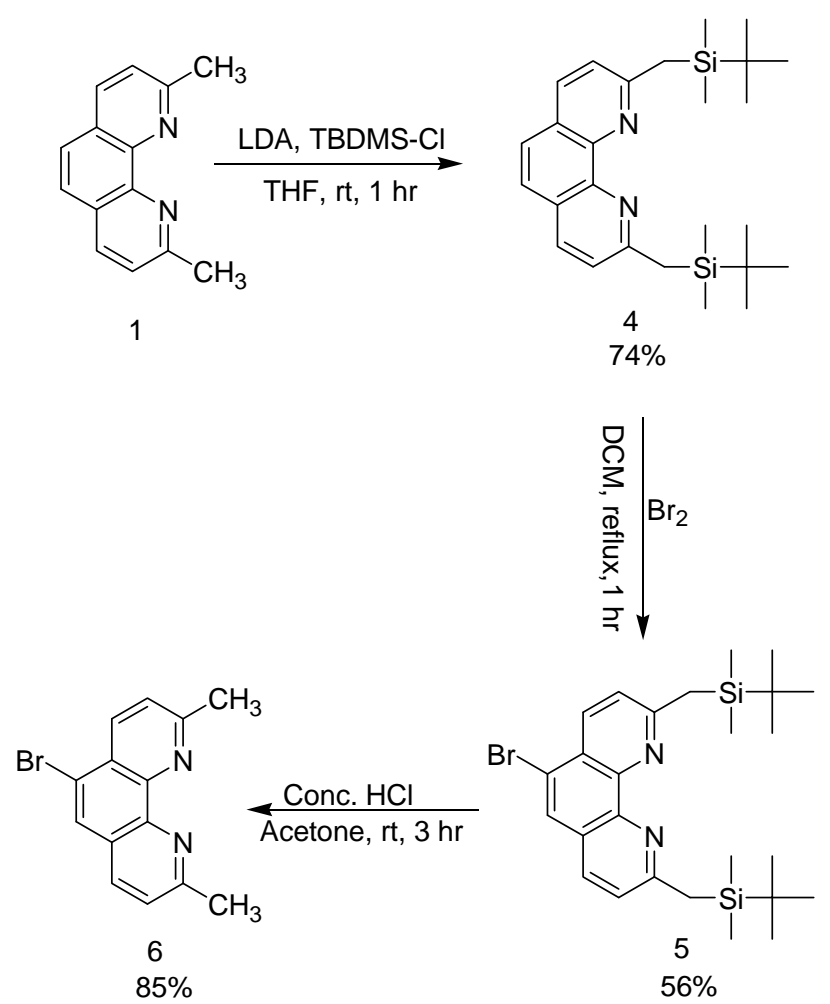

Scheme 3. Synthesis of 5-bromo-1,10-phenanthroline-2,9-dicarboxaldehyde.<smiles>Cc1ccc2cc(Br)c3ccc(S(=O)(=O)[O-])nc3c2n1</smiles>

Scheme 4. Synthesis of 5-Bromo-1, 10-Phenanthroline-2,9-dicarboxaldehyde. 


\subsection{Synthesis of the Schiff Bases with Thiosemicarbazide}

Thiosemicarbazide (4 equiv.) was added to a hot solution of 5-nitro- or 5-bromo-1,10-phenanthroline-2,9-dicarboxaldehyde ( 1 equiv) in methanol $(20 \mathrm{~mL})$ containing 2 3 drops of conc. sulfuric acid. The solution was refluxed for $1 \mathrm{hr}$ whereupon the reaction mixture was allowed to cool down to room temperature. The product that had formed was filtered off, washed with methanol and dried under vacuum. Recrystallization of the crude product from dichloromethane afforded white crystals (Scheme 5). Table 1 shows different reaction conditions and percent yields for the two starting materials.

(2,2')-2'-(5-Nitro-1,10-Phenanthroline-2,9-diyl) bis (methan-1-yl-1-ylidene)bis-(hydrazinecar-bodithioate) (8): IR: $v\left(\mathrm{~cm}^{-1}\right)$ : $3250\left(\mathrm{NH}_{2}\right), 3154(\mathrm{C}-\mathrm{H}), 1589(\mathrm{C}=\mathrm{N})$, $1522(\mathrm{~N}=\mathrm{O}), 1115(\mathrm{C}=\mathrm{S}) .{ }^{1} \mathrm{H}-\mathrm{NMR}\left(\mathrm{DMSO}_{6}, \mathrm{ppm}\right): \delta_{\mathrm{H}}$ $=12.05(\mathrm{~s}, 1 \mathrm{NH}), 12.03(\mathrm{~s}, 1 \mathrm{NH}), 9.0(\mathrm{~s}, 1 \mathrm{H}), 8.86(\mathrm{~s}$, $2 \mathrm{H}), 8.86(\mathrm{~d}, \mathrm{~J}=7.44,1 \mathrm{H}), 8.76(\mathrm{~d}, \mathrm{~J}=8.56,1 \mathrm{H}), 8.57$ (d, $\mathrm{J}=7.44 .1 \mathrm{H},), 8.48(\mathrm{~d}, \mathrm{~J}=8.56,1 \mathrm{H}), 7.60\left(\mathrm{~s}, \mathrm{br}, 2 \mathrm{NH}_{2}\right)$. ${ }^{13} \mathrm{C}-\mathrm{NMR}\left(\mathrm{DMSO}-\mathrm{d}_{6}, \mathrm{ppm}\right): \delta_{\mathrm{C}}=178.61,178.58,156.80$, $154.60,146.35,144.90,143.78,141.60,141.42,138.63$, $132.39,125.88,125.70,121.02,120.97,120.49$. LC-MS $(\mathrm{m} / \mathrm{z}): 428\left(\mathrm{M}+\mathrm{H}^{+}\right), 427 \quad\left(\mathrm{M}^{+}\right), 426\left(\mathrm{M}-\mathrm{H}^{+}\right), 411$ $\left(\mathrm{M}+\mathrm{H}^{+}-\mathrm{NH}_{3}\right), 399\left(\mathrm{M}+2 \mathrm{H}^{+}-\mathrm{N}_{2} \mathrm{H}_{2}\right), \quad 391\left(\mathrm{M}+\mathrm{H}^{+}-2 \mathrm{NH}_{2}\right)$, $383\left(\mathrm{M}+2 \mathrm{H}^{+}-\mathrm{NO}_{2}\right), \quad 336\left(\mathrm{M}+2 \mathrm{H}^{+}-\mathrm{CS}_{2}-\mathrm{NH}_{3}\right), \quad 254$ $\left(\mathrm{M}+4 \mathrm{H}^{+}-\mathrm{N}_{5} \mathrm{C}_{3} \mathrm{~S}_{2} \mathrm{H}_{7}\right)$.

(2,2')-2'-(5-Bromo-1,10-Phenanthroline-2,9-diyl)bis (methan-1-yl-1-ylidene) bis-(hydrazinecar-bodithioate) (9): IR: $v\left(\mathrm{~cm}^{-1}\right): 3300\left(\mathrm{NH}_{2}\right), 3160(\mathrm{C}-\mathrm{H}), 2951(\mathrm{CH}$ aromatic), $1568(\mathrm{C}=\mathrm{N}), 1092(\mathrm{C}=\mathrm{S}) .{ }^{1} \mathrm{H}-\mathrm{NMR}$ (DMSO$\left.\mathrm{d}_{6}, \mathrm{ppm}\right): \delta_{\mathrm{H}}=12.02(\mathrm{~s}, 1 \mathrm{NH}), 12.00(\mathrm{~s}, 1 \mathrm{NH}), 8.84(\mathrm{~d}, \mathrm{~J}$ $=8.68,1 \mathrm{H}), 8.76(\mathrm{~d}, \mathrm{~J}=8.48,1 \mathrm{H}), 8.60(\mathrm{~d}, \mathrm{~J}=8.76,1 \mathrm{H})$, $8.54(\mathrm{~s}, 1 \mathrm{H}), 8.52(\mathrm{~s}, 2 \mathrm{H}), 8.45(\mathrm{~d}, \mathrm{~J}=9.24,1 \mathrm{H}), 7.56(\mathrm{~s}$,

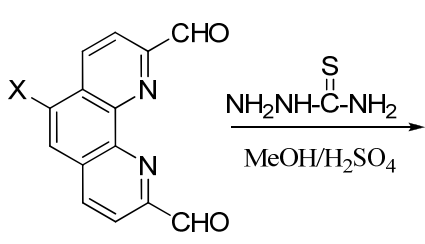

3. $\mathrm{X}=\mathrm{NO}_{2}$

7. $\mathrm{X}=\mathrm{Br}$

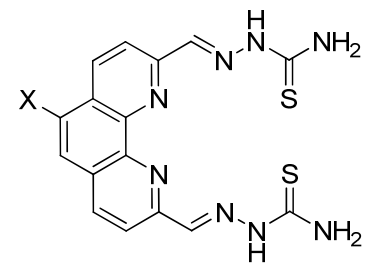

8. $\mathrm{X}=\mathrm{NO}_{2}$ 9. $\mathrm{X}=\mathrm{Br}$
Scheme 5. Synthesis of Schiff Bases with thiosemicarbazide.

Table 1. Reaction conditions and percent yields for the synthesis of the schiff bases.

\begin{tabular}{|c|c|c|c|c|}
\hline \multirow{2}{*}{ Condition } & \multirow{2}{*}{ Solvent } & \multirow{2}{*}{ Time } & \multicolumn{2}{|c|}{ Yield \% } \\
\hline & & & $\mathrm{X}=\mathrm{NO}_{2}$ & $\mathrm{X}=\mathrm{Br}$ \\
\hline With $\mathrm{H}_{2} \mathrm{SO}_{4}$ & $\mathrm{HC}_{3} \mathrm{OH}$ & $1 \mathrm{hr}$ & 89 & 81 \\
\hline $\begin{array}{l}\text { Without } \\
\mathrm{H}_{2} \mathrm{SO}_{4}\end{array}$ & $\mathrm{CH}_{3} \mathrm{OH}$ & $1 \mathrm{hr}$ & 60 & 57 \\
\hline
\end{tabular}

br, $2 \mathrm{NH}_{2}$ ). ${ }^{13} \mathrm{C}-\mathrm{NMR}$ (DMSO-d $\left.6, \mathrm{ppm}\right): \delta_{\mathrm{C}}=178.54(2)$, $154.36,154.02,144.94,143.73,141.66,141.43,136.35$, $135.73,130.19,128.99,127.28,121.02,120.74,120.11$. LC-MS (m/z): 463/461 (M+2H $\left.{ }^{+}\right), 461 / 459\left(\mathrm{M}^{+}\right), 446 / 444$ $\left(\mathrm{M}+2 \mathrm{H}^{+}-\mathrm{NH}_{3}\right), \quad 429 / 427 \quad\left(\mathrm{M}+2 \mathrm{H}^{+}-2 \mathrm{NH}_{3}\right), \quad 413 / 414$ $\left(\mathrm{M}^{+}-\mathrm{S}-\mathrm{NH}_{2}\right), 399\left(\mathrm{M}^{+}-\mathrm{NH}_{2} \mathrm{CSH}\right), 385 / 383\left(\mathrm{M}+3 \mathrm{H}^{+}-\mathrm{Br}\right)$. $349 / 350\left(\mathrm{M}^{+}-\mathrm{CS}_{2}-2 \mathrm{NH}_{3}\right), 337 / 335\left(\mathrm{M}^{+}-2 \mathrm{C}_{2} \mathrm{~S}_{2} \mathrm{H}_{2}-2 \mathrm{NH}_{3}\right)$, $281 / 279\left(\mathrm{M}+4 \mathrm{H}^{+}-2 \mathrm{CS}_{2}-2 \mathrm{NH}_{2}\right)$.

\subsection{Synthesis of the Schiff Bases with S-Methyldithiocarbazate (SMDTC):}

SMDTC was prepared following the procedure of Tarafder, et al. [11].

SMDTC (3 equiv.) was added to a hot solution of 5-substituted $\left(\mathrm{NO}_{2}, \mathrm{Br}\right)$ 1,10-phenanthroline-2,9-dicarboxaldehyde (1 equiv.) in $20 \mathrm{~mL}$ methanol containing 2 3 drops of conc. sulfuric acid. The solution was refluxed for $1 \mathrm{hr}$ and then allowed to cool to room temperature. The solid product that had formed was filtered off, washed with methanol and dried under vacuum. The crude product was recrystallized from dimethyl sulfoxide to obtain white crystals (Scheme 6). Yields are shown in Table 2 for various reaction conditions.

(2,2')-Dimethyl 2,2'-(5-Nitro-1,10-Phenanthro-line2,9-diyl)bis(methan-1-yl-1-ylidene)-bis(hydr-azinecar bodithioate)(10):

IR: $v\left(\mathrm{~cm}^{-1}\right): 3200(\mathrm{~N}-\mathrm{H}), 2980,2914(\mathrm{CH}$ aromatic and aliphatic), $1580(\mathrm{C}=\mathrm{N}), 1527(\mathrm{~N}=\mathrm{O}), 1099(\mathrm{C}=\mathrm{S})$. ${ }^{1} \mathrm{H}-\mathrm{NMR}\left(\mathrm{DMSO}-\mathrm{d}_{6}, \mathrm{ppm}\right): \delta_{\mathrm{H}}=13.78(\mathrm{~s}, 1 \mathrm{NH}), 13.77$ $(\mathrm{s}, 1 \mathrm{NH}), 9.02(\mathrm{~s}, 1 \mathrm{H}), 8.94(\mathrm{~d}, \mathrm{~J}=8.64,1 \mathrm{H}), 8.78(\mathrm{~d}, \mathrm{~J}=$ $7.96,1 \mathrm{H}), 8.52(\mathrm{~s}, 2 \mathrm{H}), 8.35(\mathrm{~d}, \mathrm{~J}=9.00,1 \mathrm{H}), 8.32(\mathrm{~d}, \mathrm{~J}=$ 8.36, 1H), $2.61\left(\mathrm{~s}, 6 \mathrm{H}, \mathrm{CH}_{3}\right) .{ }^{13} \mathrm{C}-\mathrm{NMR}$ (DMSO-d 6 , ppm): $\delta_{\mathrm{C}}=200.13(2), 155.57,153.53,146.45,145.43(2)$,
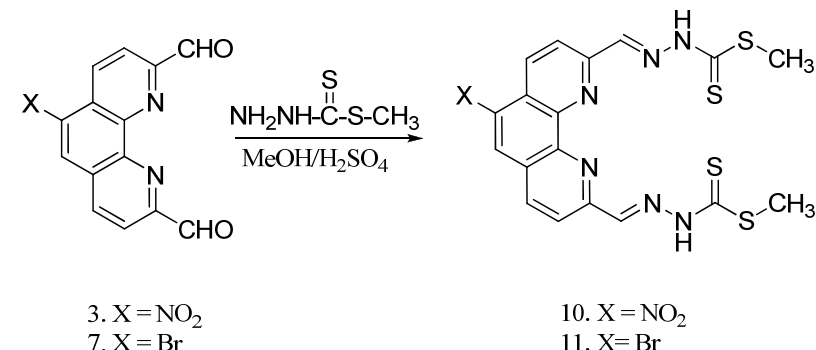

Scheme 6. Synthesis of the Schiff Bases with S-methyldithiocarbazate (SMDTC).

Table 2. Reaction conditions and percent yields for the synthesis of the schiff bases.

\begin{tabular}{ccccc}
\hline \multirow{2}{*}{ Condition } & \multirow{2}{*}{ Solvent } & Time & \multicolumn{2}{c}{ Yield \% } \\
\cline { 4 - 5 } & & & $\mathrm{X}=\mathrm{NO}_{2}$ & $\mathrm{X}=\mathrm{NO}_{2}$ \\
\hline With $\mathrm{H}_{2} \mathrm{SO}_{4}$ & $\mathrm{CH}_{3} \mathrm{OH}$ & $1 \mathrm{hr}$ & 55 & 54 \\
$\begin{array}{c}\text { Without } \\
\mathrm{H}_{2} \mathrm{SO}_{4}\end{array}$ & $\mathrm{CH}_{3} \mathrm{OH}$ & $1 \mathrm{hr}$ & 35 & 46 \\
\hline
\end{tabular}


$145.27(2), \quad 145.05,139.35,133.26,126.39,126.16$, 121.01, 120.38, 16.95(2). LC-MS $\left(\mathrm{m} / \mathrm{z}, \mathrm{M}^{+}\right): 492$ $\left(\mathrm{M}+3 \mathrm{H}^{+}\right), 491\left(\mathrm{M}+2 \mathrm{H}^{+}\right), 490\left(\mathrm{M}+\mathrm{H}^{+}\right), 489\left(\mathrm{M}^{+}\right), 474\left(\mathrm{M}^{+}-\right.$ $\left.\mathrm{CH}_{3}\right), \quad 459\left(\mathrm{M}^{+}-2 \mathrm{CH}_{3}\right), 443\left(\mathrm{M}^{+}-\mathrm{NO}_{2}\right), 397\left(\mathrm{M}+2 \mathrm{H}^{+}-\right.$ $\left.2 \mathrm{CH}_{3} \mathrm{~S}\right), \quad 384 \quad\left(\mathrm{M}+\mathrm{H}^{+}-\mathrm{CH}_{3} \mathrm{SCSNH}\right), \quad 308 \quad\left(\mathrm{M}+\mathrm{H}^{+}-\right.$ $\left.2 \mathrm{CH}_{3} \mathrm{SCS}\right), \quad 307 \quad\left(\mathrm{M}^{+}-2 \mathrm{CH}_{3} \mathrm{SCS}\right), \quad 303 \quad\left(\mathrm{M}^{+}-2 \mathrm{CH}_{3} \mathrm{SH}-\right.$ $\mathrm{C}_{2} \mathrm{H}_{2} \mathrm{~S}_{2}$ ).

(2,2')-Dimethyl 2,2'-(5-Bromo-1,10-Phenanthro-line2,9-diyl)bis(methan-1-yl-1-ylidene)-bis(hydr-azinecar bodithioate)(11):

IR: $v\left(\mathrm{~cm}^{-1}\right): 3240(\mathrm{~N}-\mathrm{H}), 3160,2915(\mathrm{CH}$ aromatic and aliphatic), $1567 \quad(\mathrm{C}=\mathrm{N}), \quad 1056(\mathrm{C}=\mathrm{S}) .{ }^{1} \mathrm{H}-\mathrm{NMR}$ $\left(\mathrm{DMSO}_{-} \mathrm{d}_{6}, \mathrm{ppm}\right): \delta_{\mathrm{H}}=13.79(\mathrm{~s}, 1 \mathrm{NH}), 13.76(\mathrm{~s}, 1 \mathrm{NH})$, $8.74(\mathrm{~d}, \mathrm{~J}=8.60,1 \mathrm{H}), 8.61(\mathrm{~s}, 1 \mathrm{H}), 8.57(\mathrm{~s}, 2 \mathrm{H}), 8.56(\mathrm{~d}$, $\mathrm{J}=8.56,1 \mathrm{H}), 8.39(\mathrm{~d}, \mathrm{~J}=8.84,1 \mathrm{H}), 8.29(\mathrm{~d}, \mathrm{~J}=8.40,1 \mathrm{H})$, $2.61\left(\mathrm{~s}, \quad 3 \mathrm{H}, \quad \mathrm{CH}_{3}\right), 2.60 \quad\left(\mathrm{~s}, \quad 3 \mathrm{H}, \mathrm{CH}_{3}\right) .{ }^{13} \mathrm{C}-\mathrm{NMR}$ $\left(\right.$ DMSO-d $\left._{6}, \mathrm{ppm}\right): \delta_{\mathrm{C}}=199.89,199.77,153.30,153.07$, 145.87, 145.46, 144.34, 136.72, 136.68, 130.73, 130.72, $129.37,127.93,120.70,120.31,119.91,16.93(2)$. LC-MS $\left(\mathrm{m} / \mathrm{z}, \mathrm{M}^{+}\right)$: 525/523 $\left(\mathrm{M}+\mathrm{H}^{+}\right), 524 / 522\left(\mathrm{M}^{+}\right)$, $477 / 475\left(\mathrm{M}^{+}-\mathrm{CH}_{3} \mathrm{~S}\right), 450 / 448\left(\mathrm{M}+2 \mathrm{H}^{+}-\mathrm{CS}_{2}\right), 443 / 441$ $\left(\mathrm{M}^{+}-\mathrm{Br}\right), 429 / 427\left(\mathrm{M}^{+}-2 \mathrm{CH}_{3} \mathrm{~S}\right), 366\left(\mathrm{M}+\mathrm{H}^{+}-\mathrm{Br}^{-} \mathrm{CS}_{2}\right), 338$ $\left(\mathrm{M}^{+}-\mathrm{Br}-\mathrm{CH}_{3} \mathrm{~S}-2 \mathrm{~N}_{2}\right)$.

\subsection{Synthesis of the Schiff Bases with S-Benzyldithiocarbazate (SBDTC)}

SBDTC was prepared using a procedure described by Audrieth et al. [12]. SBDTC (3 equiv) was added to a hot solution of 5-substituted $\left(\mathrm{NO}_{2}, \mathrm{Br}\right)$ 1,10-phenanthroline2,9-dicarboxaldehyde ( 1 equiv.) in $20 \mathrm{~mL}$ methanol followed by the addition of two drops of acetic acid. The reaction mixture was refluxed for $1 \mathrm{~h}$, then left to cool to room temperature. The product that had formed was filtered off, washed with methanol and dried in vacuum. White crystals of the compound were obtained by recrystallizing the crude product from dimethylsulfoxide (Scheme 7). Yields with and without acid are given in Table 3.

(2,2')-Benzyl 2,2'-(5-Nitro-1,10-Phenanthroline-2,9diyl)bis(methan-1-yl-1-ylidene)-bis(hydrazinecarbodit hioate)(12): IR: $v\left(\mathrm{~cm}^{-1}\right)$ : $3150(\mathrm{~N}-\mathrm{H}), 3026(\mathrm{C}-\mathrm{H}$ aromatic), 2920 (C-H aliphatic), $1566(\mathrm{C}=\mathrm{N}), 1605,1494$ $(\mathrm{C}=\mathrm{C}$ aromatic $), 1526 \quad(\mathrm{~N}=\mathrm{O}), \quad 1094(\mathrm{C}=\mathrm{S}) .{ }^{1} \mathrm{H}-\mathrm{NMR}$ $\left(\mathrm{DMSO}^{\mathrm{d}} \mathrm{d}_{6}, \mathrm{ppm}\right): \delta_{\mathrm{H}}=13.79(\mathrm{~s}, 1 \mathrm{NH}), 13.78(\mathrm{~s}, 1 \mathrm{NH})$, $8.99(\mathrm{~s}, 1 \mathrm{H}), 8.88(\mathrm{~d}, \mathrm{~J}=8.80,1 \mathrm{H}), 8.71(\mathrm{~d}, \mathrm{~J}=8.32,1 \mathrm{H})$, $8.48(\mathrm{~s}, 1 \mathrm{H}), 8.47(\mathrm{~s}, 1 \mathrm{H}), 8.29(\mathrm{~d}, \mathrm{~J}=8.88,1 \mathrm{H}), 8.25(\mathrm{~d}, \mathrm{~J}=$ 8.44, 1H), $7.48-7.31(\mathrm{~m}, 10 \mathrm{H}), 4.54\left(\mathrm{~s}, 4 \mathrm{H}, 2 \mathrm{xCH}_{2}\right)$. ${ }^{13} \mathrm{C}-\mathrm{NMR}\left(\mathrm{DMSO}-\mathrm{d}_{6}, \mathrm{ppm}\right): \delta_{\mathrm{C}}=198.25,198.14,155.51$, $153.41,146.41,145.62,139.36,136.38,129.32(6)$, $129.12(8), 128.56(2), 127.38,126.19,121.03,120.39$, 37.87(2). LC-MS (m/z): $642 \quad\left(\mathrm{M}+\mathrm{H}^{+}\right), 641 \quad\left(\mathrm{M}^{+}\right)$, $597\left(\mathrm{M}+\mathrm{H}^{+}-\mathrm{NO}_{2}\right), 479\left(\mathrm{M}+3 \mathrm{H}^{+}-\mathrm{PhCH}_{2} \mathrm{~S}\right), 393\left(\mathrm{M}+2 \mathrm{H}^{+}-\right.$ $\left.2 \mathrm{PhCH}_{2} \mathrm{~S}\right), 356\left(\mathrm{M}+4 \mathrm{H}^{+}-2 \mathrm{PhCH}_{2}-\mathrm{CS}_{2}-\mathrm{S}\right), 303\left(\mathrm{M}+2 \mathrm{H}^{+}-\right.$ $\left.2 \mathrm{PhCH}_{2} \mathrm{SCS}\right), 279\left(\mathrm{M}+2 \mathrm{H}^{+}-2 \mathrm{PhCH}_{2} \mathrm{SCSNH}\right)$.

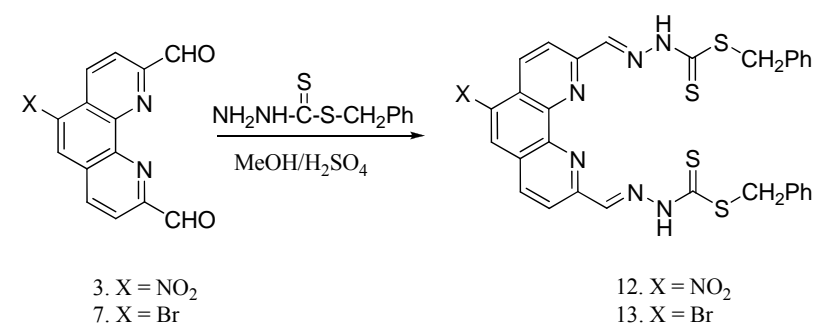

Scheme 7. Synthesis of the Schiff Bases with S-benzyldithiocarbazate (SBDTC).

Table 3. Reaction Conditions and Percent Yields for the Synthesis of Schiff Bases.

\begin{tabular}{ccccc}
\hline \multirow{2}{*}{ Condition } & \multirow{2}{*}{ Solvent } & \multirow{2}{*}{ Time } & \multicolumn{2}{c}{ Yield \% } \\
\cline { 4 - 5 } & & & $\mathrm{X}=\mathrm{NO}_{2}$ & $\mathrm{X}=\mathrm{NO}_{2}$ \\
\hline With $\mathrm{H}_{2} \mathrm{SO}_{4}$ & $\mathrm{CH}_{3} \mathrm{OH}$ & $1 \mathrm{hr}$ & 65 & 96 \\
$\begin{array}{c}\text { Without } \\
\mathrm{H}_{2} \mathrm{SO}_{4}\end{array}$ & $\mathrm{CH}_{3} \mathrm{OH}$ & $1 \mathrm{hr}$ & 60 & 92 \\
\hline
\end{tabular}

(2,2')-Benzyl 2,2'-(5-Bromo-1,10-Phenanthro line2,9-diyl)bis(methan-1-yl-1-ylidene)-bis(hydrazinecarb odithioate)(13):

IR: $v\left(\mathrm{~cm}^{-1}\right): 3180(\mathrm{~N}-\mathrm{H}), 3027(=\mathrm{CH}$ aromatic), 2925 (C-H aliphatic), $1566(\mathrm{C}=\mathrm{N}), 1600,1479(\mathrm{C}=\mathrm{C}$ aromatic $)$, $1095(\mathrm{C}=\mathrm{S}) .{ }^{1} \mathrm{H}-\mathrm{NMR}\left(\mathrm{DMSO}-\mathrm{d}_{6}, \mathrm{ppm}\right): \delta_{\mathrm{H}}=13.76(\mathrm{~s}$, $1 \mathrm{NH}), 13.73(\mathrm{~s}, 1 \mathrm{NH}), 8.58(\mathrm{~d}, \mathrm{~J}=8.24,1 \mathrm{H}), 8.51(\mathrm{~s}, 1 \mathrm{H})$, $8.49(\mathrm{~s}, 1 \mathrm{H}), 8.43(\mathrm{~s}, 1 \mathrm{H}), 8.41(\mathrm{~d}, \mathrm{~J}=8.42,1 \mathrm{H}), 8.24(\mathrm{~d}$, $\mathrm{J}=8.36,1 \mathrm{H}), 8.15(\mathrm{~d}, \mathrm{~J}=7.96,1 \mathrm{H}), 7.48-7.27(\mathrm{~m}, 10 \mathrm{H})$, $4.54(\mathrm{~s}, 4 \mathrm{H}) .{ }^{13} \mathrm{C}-\mathrm{NMR}\left(\mathrm{DMSO}-\mathrm{d}_{6}, \mathrm{ppm}\right): \delta_{\mathrm{C}}=198.04(2)$, $153.35,153.30,146.28,145.13,143.59,136.74,136.46$, 129.29(6), 129.08(8), 129.56(2), 128.33, 127.37, 120.70, 52.69, 52.79. LC-MS (m/z): 677/675 $\left(\mathrm{M}+\mathrm{H}^{+}\right), 676 / 674$ $\left(\mathrm{M}^{+}\right), 597 / 595\left(\mathrm{M}^{+}-\mathrm{Br}\right), 553 / 551\left(\mathrm{M}^{+}-\mathrm{PhCH}_{2} \mathrm{~S}\right), 526 / 524$ $\left(\mathrm{M}^{+}-2 \mathrm{CH}_{3} \mathrm{~S}-2 \mathrm{~N}_{2}\right), 403 / 401\left(\mathrm{M}+\mathrm{H}^{+}-2 \mathrm{PhCH}_{2} \mathrm{~S}-\mathrm{N}_{2}\right), 391$ $\left(\mathrm{M}+4 \mathrm{H}^{+}-\mathrm{Br}-2 \mathrm{PhCH}_{2}-\mathrm{N}_{2}\right)$.

\subsection{Synthesis of the Schiff Bases with 2-Mercaptoaniline}

The compound was prepared using the same procedure as described in 3.3, using 2-mercaptoaniline (4 equiv.) and 5-substituted $\left(\mathrm{NO}_{2}, \mathrm{Br}\right)$ 1,10-phenanthroline-2,9-dicarboxaldehyde (1 equiv.). Table 4 shows different reaction conditions and percent yields. Cyclized products were obtained in both cases as shown in the equation (Scheme 8).

2,9-Di-(benzo[d]thiazol-2-yl)-5-Nitro-1,10-Phenanthroline (14): IR: $v\left(\mathrm{~cm}^{-1}\right): 3062(\mathrm{CH}$ aromatic), 1610 $(\mathrm{C}=\mathrm{C}$ aromatic $), 1579(\mathrm{C}=\mathrm{N}), 1555(\mathrm{C}=\mathrm{C}) 1528(\mathrm{~N}=\mathrm{O})$. ${ }^{1} \mathrm{H}-\mathrm{NMR}\left(\right.$ DMSO- $_{6}$ ) $): \delta_{\mathrm{H}}=8.89(\mathrm{~s}, 1 \mathrm{H}), 8.83(\mathrm{~d}, \mathrm{~J}=7.28$, $1 \mathrm{H}), 8.68(\mathrm{~d}, \mathrm{~J}=7.56,1 \mathrm{H}), 8.61(2 \mathrm{~d}$, app t, $\mathrm{J}=8.76,2 \mathrm{H})$, $8.33-8.23(\mathrm{~m}, 4 \mathrm{H}), 7.64(\mathrm{~m}, 4 \mathrm{H}) .{ }^{13} \mathrm{C}-\mathrm{NMR}$ (DMSO-d ${ }_{6}$, ppm): $\delta_{\mathrm{C}}=168.77,168.60,154.89,154.89,153.99$, $152.07,149.23,147.15145 .54,144.20,136.36(2)$, $136.21,134.30,126.90(2), 126.72(3), 126.40,126.33$, 

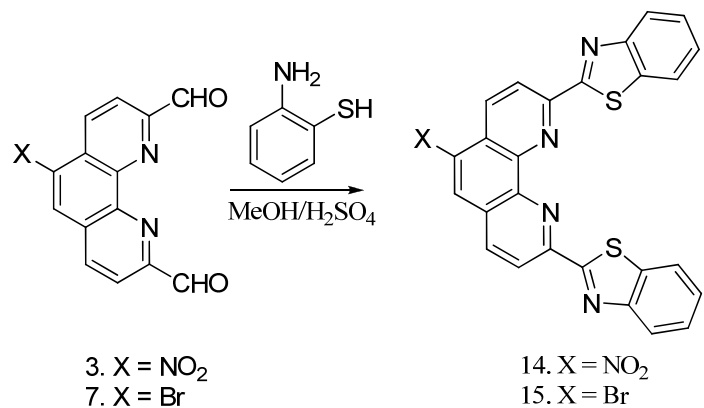

14. $\mathrm{X}=\mathrm{NO}_{2}$

7. $\mathrm{X}=\mathrm{Br}$

15. $\mathrm{X}=\mathrm{Br}$

Scheme 8. Synthesis of Substituted Schiff Bases with 2-Marcaptoaniline

Table 4. Reaction Conditions and Percent Yields of the Schiff Bases.

\begin{tabular}{ccccc}
\hline \multirow{2}{*}{ Condition } & Solvent & Time & \multicolumn{2}{c}{ Yield \% } \\
\cline { 4 - 5 } & & & $\mathrm{X}=\mathrm{NO}_{2}$ & $\mathrm{X}=\mathrm{NO}_{2}$ \\
\hline With $\mathrm{H}_{2} \mathrm{SO}_{4}$ & $\mathrm{CH}_{3} \mathrm{OH}$ & $1 \mathrm{hr}$ & 45 & 30 \\
$\begin{array}{c}\text { Without } \\
\mathrm{H}_{2} \mathrm{SO}_{4}\end{array}$ & $\mathrm{CH}_{3} \mathrm{OH}$ & $1 \mathrm{hr}$ & 25 & 25 \\
\hline
\end{tabular}

$123.85,123.67,122.80 .121 .89,121.09$. LC-MS (m/z, $\left.\mathrm{M}^{+}\right): 494\left(\mathrm{M}+3 \mathrm{H}^{+}\right), 492\left(\mathrm{M}+2 \mathrm{H}^{+}\right), 491\left(\mathrm{M}^{+}\right), 462\left(\mathrm{M}+\mathrm{H}^{+}-\right.$ $\mathrm{NO}), 447 \quad\left(\mathrm{M}+2 \mathrm{H}^{+}-\mathrm{NO}_{2}\right), 391\left(\mathrm{M}+2 \mathrm{H}^{+}-\mathrm{C}_{6} \mathrm{H}_{4} \mathrm{CN}\right), 392$ $\left(\mathrm{M}+3 \mathrm{H}^{+}-\mathrm{C}_{6} \mathrm{H}_{4} \mathrm{CN}\right), 359\left(\mathrm{M}+2 \mathrm{H}^{+}-\mathrm{C}_{6} \mathrm{H}_{4} \mathrm{CNS}\right), 283\left(\mathrm{M}^{+}-\right.$ $\left.2 \mathrm{C}_{6} \mathrm{H}_{4} \mathrm{CN}\right), 254\left(\mathrm{M}+3 \mathrm{H}^{+}-2 \mathrm{C}_{6} \mathrm{H}_{4} \mathrm{CS}\right)$.

2,9-Di-(benzo[d]thiazol-2-yl)-5-Bromo-1,10-Phenan throline (15): IR: $v\left(\mathrm{~cm}^{-1}\right): 3053$ (CH aromatic), 1602 $(\mathrm{C}=\mathrm{C}$ aromatic $), 1579(\mathrm{C}=\mathrm{N}), 1547(\mathrm{C}=\mathrm{C}) .{ }^{1} \mathrm{H}-\mathrm{NMR}$ $\left(\right.$ DMSO-d $_{6}$ ) $: \delta_{\mathrm{H}}=8.87$ (3d,app q, $\left.\mathrm{J}=8.4,3 \mathrm{H}\right), 8.75(\mathrm{~s}$, $1 \mathrm{H}), 8.71(\mathrm{~d}, \mathrm{~J}=7.47,1 \mathrm{H}), 8.35-8.22(\mathrm{~m}, 4 \mathrm{H}), 7.64(\mathrm{~m}$, 4H). ${ }^{13} \mathrm{C}-\mathrm{NMR}$ (DMSO-d 6, ppm): $\delta_{\mathrm{C}}=169.07(2)$, 153.93(2), 149.67, 137.44(2), 136.19(2), 135.37(2), $131.13(2), 130.98,126.83(2), 126.53,123.68,122.78$, 120.33(2), 119.57, 116.43(2), 116.09, 114.79. LC-MS $\left(\mathrm{m} / \mathrm{z}, \mathrm{M}^{+}\right): 528 / 526\left(\mathrm{M}+2 \mathrm{H}^{+}\right), 527 / 525\left(\mathrm{M}+\mathrm{H}^{+}\right), 526 / 524$ $\left(\mathrm{M}^{+}\right), 474 / 472\left(\mathrm{M}^{+}-2 \mathrm{CN}\right), 452 / 450\left(\mathrm{M}+2 \mathrm{H}^{+}-\mathrm{CS}_{2}\right), 391$ $\left(\mathrm{M}+\mathrm{H}^{+}-\mathrm{C}_{6} \mathrm{H}_{4} \mathrm{CNS}\right), 279\left(\mathrm{M}^{+}-\mathrm{C}_{6} \mathrm{H}_{4} \mathrm{CH}_{2} \mathrm{~S}\right)$.

\section{Conclusion}

Eight new Schiff bases of 5-nitro- and 5-bromo-2,9-dimethyl-1,10-phenanthroline dialdehydes with sufur-containing amines have been successfully synthesized. The addition of conc. $\mathrm{H}_{2} \mathrm{SO}_{4}$ to the reaction mixtures has a significant effect on the yields of the products. We tried to do the reaction without acid because of the possibility of forming salts by the protonation of nitrogen atoms of the phenanthroline moiety which might cause lower solubility of the Schiff bases. However, it was observed that the yield increased significantly when the reaction was carried out under mild aicidic conditions. This is due to the fact that protonation of the carbonyl group $(\mathrm{C}=\mathrm{O})$ enhances the nucleophilic attack by an anion. Also, reac- tion time was reduced significantly compared to that reported in the previous work, probably because of the presence of the substituted group in the 5-position in the phenanthroline moiety.

\section{Acknowledgements}

We thank the Department of Chemistry at Tennessee State University for providing the necessary support to carry out the research. We also thank the Department of Education, Title III funds for providing instrumental support.

\section{REFERENCES}

[1] P. G. Sammes and G. Yahioglu, "1,10-Phenanthroline: A Versatile Ligand," Chemical Society Reviews, Vol. 23 , No. 5, 1994, pp. 327-334. doi:10.1039/cs9942300327

[2] R. Sahu, D. S. Thakur and P. Kashyap, "Schiff Base: An Overview of Its Medicinal Chemistry Potential of New Drug Molecules," International Journal of Pharmaceutical Science and Nanotechnology, Vol. 5, No. 3, 2012, pp. 1757-1764.

[3] J. P. W. Eggert, U. Lüning and C. Näther, "Synthesis and Functionalisation of 5-Substituted Neocuproine Derivatives," European Journal of Organic Chemistry, Vol. 2005, No. 6, 2005, pp. 1107-1112. doi:10.1002/ejoc.200400635

[4] J. Reedijk, "Comprehensive Coordination Chemistry," In: G. Wilkinson, R. D. Gillard and J. A. McCleverty, Eds., Comprehensive Coordination Chemistry, Pergamon, Oxford, Vol. 2, 1987, p. 73.

[5] M. Akbar Ali, A. H. Mirza, A. L. Tan, L. K. Wei and P. V. Bernhardt, "The Preparation and Characterization of Seven-Coordinated Tin(IV) Complexes of the 2,6-Diacetylpyridine Schiff Bases of S-Alkyl/Aryl-dithiocarbazates and the X-ray Crystal Structure of the [Sn(dapsme)I2] Complex (dapsme=doubly protonated form of the 2,6-Diacetylpyridine Schiff Bases of S-Methyldithiocarbazate)," Polyhedron, Vol. 23, No. 11, 2004, pp. 2037 2043. doi:10.1016/j.poly.2004.05.010

[6] M. Arifuzzaman, M. R. Karim, T. A. Siddiquee1, A. H. Mirza and M. A. Ali, "Synthesis and Characterization of New Schiff Bases Formed by Condensation of 2,9-Phenathroline-1,10-Dialdehyde with Sulfur-Containing Amines" International Journal of Organic Chemistry, Vol. 3, No. 1, 2013, pp. 81-86. doi:10.4236/ijoc.2013.31009

[7] G. F. Smith and F. W. Cagl Jr., "The Improved Synthesis of 5-Nitro-1,10-Phenanthroline," Journal Organic Chemistry, Vol. 12, No. 6, 1947, pp. 781-784. doi:10.1021/jo01170a007

[8] H. Astroem and R. Stroemberg, "Synthesis of New OBAN's and Further Studies on Positioning of the Catalytic Group," Organic \& Biomolecular Chemistry, Vol. 2, No. 13, 2004, pp. 1901-1907. doi:10.1039/b403652b

[9] R. C. Blake II, A. R. Pavlov, M. Khosraviani, H. E. Ensley, G. E. Kiefer, H. Yu, X, Li and D. A. Blake, "Novel Monoclonal Antibodies with Specificity for Chelated 
Uranium(VI): Isolation and Binding Properties," Bioconjugate Chemistry, Vol. 15, No. 5, 2004, pp. 1125-1136. doi:10.1021/bc049889p

[10] J. P. W. Eggert and U. Lüning, "Towards A,D-(1,10-Phenanthroline)-Bridged Calix[6]arene Dendrimers," European Journal of Organic Chemistry, Vol. 2007, No. 36, 2007, pp. 6046-6052. doi:10.1002/ejoc. 200700639

[11] M. T. H. Tarafder, K. B. Chew, K. A. Crouse, A. S. M. Ali, B. M. Yamin and H. K. Fun, "Synthesis and Characterization of $\mathrm{Cu}(\mathrm{II}), \mathrm{Ni}(\mathrm{II})$ and $\mathrm{Zn}$ (II) Metal complexes of Bidentate NS Isomeric Schiff Bases Derived from S-Methyldithiocarbazate (SMDTC): Bioactivity of the Biden- tate NS Isomeric Schiff bases, Some of Their $\mathrm{Cu}(\mathrm{II})$, $\mathrm{Ni}(\mathrm{II})$ and $\mathrm{Zn}$ (II) Complexes and the X-ray Structure of the Bis[S-methyl- $\beta$-N-(2-furylmethyl) Methylenedithiocarbazato]Zinc(II) Complex," Polyhedron, Vol. 21, No. 27-28, 2002, pp. 2683-2690. doi:10.1016/S0277-5387(02)01285-8

[12] Mughrabi, et al., "Cytoprotective Effect of Benzyl-N'(indol-3-ylmethylidene)-Hyrazinecarbodithioate against Ethanol-Induced Gastric Mucosal Injury in Rats," Journal of Pure and Applied Chemistry, Vol. 5, No. 3, 2011, pp. $34-42$. 